The Annals of Probability

2002, Vol. 30, No. 4, 1657-1680

\title{
POISSON APPROXIMATION FOR EXPECTATIONS OF UNBOUNDED FUNCTIONS OF INDEPENDENT RANDOM VARIABLES ${ }^{1}$
}

\author{
BY I. S. BORISOV AND P. S. RUZANKIN \\ Sobolev Institute of Mathematics of the Russian Academy of Sciences \\ Under minimal moment conditions complete asymptotic expansions \\ are obtained for expectations of unbounded functions of a finite family of \\ independent random variables in the Poissonian setting when the distributions \\ of the random variables have large atoms at zero.
}

1. Introduction and the main results. Accuracy of Poisson approximation for sums of independent random variables (r.v.s) has already been investigated for about half a century. One of the first results was obtained by Prohorov (1953), who estimated the total variation distance between a binomial distribution and the corresponding Poisson law. His upper bound is close to being unimprovable. Le Cam (1960) considerably generalized and strengthened the Prohorov estimate. He used the so-called operator technique to extend the estimate to the case of approximating distributions of sums of independent arbitrarily distributed r.v.s by corresponding generalized (accompanying) Poisson laws. Take note also of the remarkable result due to Barbour and Hall (1984) which, in particular, proved unimprovability of the Prohorov-Le Cam estimate for the total variation distance between a binomial distribution and the corresponding Poisson law.

Approximation of the next orders in the Poisson limit theorem, called an asymptotic expansion, originates from Chen (1975). Note also the paper by Deheuvels and Pfeifer (1986), where the first term of the expansion was explicitly written out. Kruopis (1986a, b) simultaneously obtained a similar result. However, he represented the first term of the expansion implicitly as the Fourier transform of some signed measure. Borovkov (1988) proposed a new approach to derive terms of the expansion in a form similar to that of Kruopis (1986a, b). He used a combination of the operator technique and a coupling to derive complete asymptotic expansion in the Poisson theorem. As in Kruopis (1986a, b) the expansion was presented via the Fourier transforms and, moreover, a scheme to invert the transforms was discussed. However, in our opinion, the resulting explicit representation of the terms of the asymptotic expansion is resistant to analysis [in contrast, say, to Deheuvels and Pfeifer (1986)].

Received November 2000; revised November 2001.

${ }^{1}$ Supported in part by the Russian Foundation for Basic Research Grants 00-01-00802 and 9901-00504 and INTAS Grant 99-01317.

AMS 2000 subject classifications. 60F05, 60E07.

Key words and phrases. Poisson approximation, asymptotic expansions, the Lindeberg method. 
It is worth noting that in all of the above-mentioned papers remainders of the expansions are estimated in terms of the total variation distance. Thus, it is clear that these results can be reformulated in terms of moments of bounded functions with the corresponding upper bounds of remainders taken uniformly over all bounded (say, by 1) functions. There are also more publications where Poisson approximation of the moments is considered uniformly over special subclasses of bounded functions [e.g., see Roos $(1995,1998)]$.

However, the total variation distance, the point metric and some related distances become unsuitable for approximation of moments of unbounded functions of a binomial r.v. In this connection, investigations of the Poisson approximation for expectations of unbounded functions of sums of r.v.s are to be distinguished. The papers which are to be particularly noted are those by Barbour (1987) and Barbour, Chen and Choi (1995). Barbour (1987) used the so-called Stein-Chen method to obtain complete asymptotic expansions in the Poisson approximation of at most polynomially growing functions of sums of independent arbitrarily distributed integer nonnegative r.v.s. In the latter paper, a similar approach is used to obtain the first term of the expansion for expectation of some functions of a binomial r.v. under minimal moment restrictions on the functions under consideration. In particular, these restrictions allow the functions growing faster than exponential. The bounds on the remainders of the expansions in these two papers are not far from optimal.

The main goal of the present paper is to obtain complete asymptotic expansions of moments of unbounded functions of $n$ independent r.v.s in the case when each of these r.v.s is equal to zero with high probability. The probabilities for these r.v.s to be unequal to zero are considered as natural small parameters, sums of which powers are used to represent the asymptotic expansions. In the case of asymptotic expansions for bounded functions a similar formulation of the problem was given by Borovkov (1988). The particular case, wherein the functions depend only on the sum of the r.v.s, is separately studied. In this case the estimate of the remainder of the expansion is unimprovable in some sense and improves the corresponding results of Barbour (1987) and Barbour, Chen and Choi (1995) in a broad range of change of the expansion parameters.

The approach used to derive the main results is based on the so-called Lindeberg method. This method was successfully employed in a great number of papers to study rates of convergence in the central limit theorem (the Gaussian case) under various settings including studying remainders in different asymptotic expansions. However, the method was rarely used in the Poisson approximation. One can find certain versions of the Lindeberg method in the Poisson approximation, for example, in the papers by Le Cam (1960), Deheuvels, Karr, Pfeifer and Serfling (1988) and Novak (1998).

In Section 5 it is shown that the problem of the Poisson approximation for expectation of a function of independent arbitrarily distributed r.v.s can be reduced 
to the case of independent Bernoulli r.v.s. Thus, in the present paper, this case is particularly studied.

Let $\zeta_{1}, \ldots, \zeta_{n}$ be independent Bernoulli r.v.s with the success probabilities $p_{j}=\mathbf{P}\left(\zeta_{j}=1\right), j=1, \ldots, n$. Let $\eta_{1}, \ldots, \eta_{n}$ be independent Poisson r.v.s with parameters $p_{1}, \ldots, p_{n}$, respectively. Denote $\lambda_{k}=p_{1}+\cdots+p_{k}, \lambda=\lambda_{n}$, and $\bar{\zeta}=\left(\zeta_{1}, \ldots, \zeta_{n}\right), \bar{\eta}=\left(\eta_{1}, \ldots, \eta_{n}\right)$. Finally, let $F$ be an arbitrary real function of $n$ nonnegative integer variables.

Denote by $\overline{e_{k}}$ the $n$-dimensional vector which has the $k$ th coordinate 1 and all the other coordinates 0 . For any function $G$ of $n$ arguments, define the difference operator $\Delta_{k}$ :

$$
\Delta_{k} G(\bar{a})=G\left(\bar{a}+\overline{e_{k}}\right)-G(\bar{a}) .
$$

In the sequel we denote by $\Delta_{k}^{r}$ the corresponding operator power. In the onedimensional case the subscript will be omitted.

The following theorem is the key result for deriving most of the subsequent statements.

Theorem 1. Suppose $\mathbf{E}|F(\bar{\eta})|<\infty$. Then

$$
\mathbf{E} F(\bar{\eta})-\mathbf{E} F(\bar{\zeta})=\sum_{k=1}^{n} \sum_{r=2}^{\infty} \frac{p_{k}^{r}}{r !} \mathbf{E} \Delta_{k}^{r} F\left(\bar{\phi}_{k}\right),
$$

where $\bar{\phi}_{k}=\left(\zeta_{1}, \ldots, \zeta_{k-1}, 0, \eta_{k+1}, \ldots, \eta_{n}\right)$, and, for each $k$, the corresponding series in (1) absolutely converges.

Moreover, if $\mathbf{E} \eta_{k}{ }^{s+1}|F(\bar{\eta})|<\infty$ for all $k$ and some $s \geq 1$, then, first, the remainder of series in (1) can be estimated as follows:

$$
\left|\sum_{r=s+1}^{\infty} \frac{p_{k}^{r}}{r !} \mathbf{E} \Delta^{r} F\left(\bar{\phi}_{k}\right)\right| \leq e^{p_{k}} \frac{p_{k}^{s+1}}{(s+1) !} \mathbf{E}\left|\Delta_{k}^{s+1} F\left(\bar{\psi}_{k}\right)\right|,
$$

where $\bar{\psi}_{k}=\bar{\phi}_{k}+\eta_{k} \bar{e}_{k}=\left(\zeta_{1}, \ldots, \zeta_{k-1}, \eta_{k}, \ldots, \eta_{n}\right)$ and, second, another expansion of the difference $\mathbf{E} F(\bar{\eta})-\mathbf{E} F(\bar{\zeta})$ holds:

$$
\begin{aligned}
& \left|\mathbf{E} F(\bar{\eta})-\mathbf{E} F(\bar{\zeta})-\sum_{r=2}^{s}(-1)^{r}(r-1) \sum_{k=1}^{n} \frac{p_{k}^{r}}{r !} \mathbf{E} \Delta_{k}^{r} F\left(\bar{\psi}_{k}\right)\right| \\
& \leq \frac{s}{(s+1) !} \sum_{k=1}^{n} e^{p_{k}} p_{k}^{s+1} \mathbf{E}\left|\Delta_{k}^{s+1} F\left(\bar{\psi}_{k}\right)\right| .
\end{aligned}
$$

REMARK 1. Under the moment restrictions considered above expansion (3) cannot be represented as a converging series with an upper bound for its remainder like in (1) and (2), because this representation would require considerably stronger moment restrictions. 
REMARK 2. The right-hand sides of the inequalities (2) and (3) can be bounded through expectations of functions of $\bar{\eta}$ using the obvious unimprovable upper bound for the Radon-Nikodym derivative of the distribution of $\bar{\psi}$ with respect to the distribution of $\bar{\eta}$ :

$$
\mathbf{E}\left|\Delta_{k}^{s+1} F\left(\bar{\psi}_{k}\right)\right| \leq e^{\lambda_{k}} \mathbf{E}\left|\Delta_{k}^{s+1} F(\bar{\eta})\right| .
$$

The right-hand side of the inequality is finite if $\mathbf{E} \eta_{k}^{s+1}|F(\bar{\eta})|<\infty$ for all $k$, since the following proposition is true:

PROPOSITION 1. Let $\tau$ be an arbitrary Poisson r.v. and $g$ be an arbitrary real function. Then, for each $l=1,2, \ldots$, the following three conditions are equivalent:

(a) $\mathbf{E} \tau^{l}|g(\tau)|<\infty$

(b) $\mathbf{E}|g(\tau+l)|<\infty$.

(c) $\mathbf{E}\left|\Delta^{l} g(\tau)\right|<\infty$.

COROLlary 1. If, for all $k, \mathbf{E} \eta_{k}{ }^{2}|F(\bar{\eta})|<\infty$, then

$$
\begin{aligned}
|\mathbf{E} F(\bar{\zeta})-\mathbf{E} F(\bar{\eta})| & \leq \frac{1}{2} \sum_{k=1}^{n} e^{p_{k}} p_{k}^{2} \mathbf{E}\left|\Delta_{k}^{2} F\left(\bar{\psi}_{k}\right)\right| \\
& \leq \frac{1}{2} \sum_{k=1}^{n} e^{\lambda_{k}} p_{k}^{2} \mathbf{E}\left|\Delta_{k}^{2} F(\bar{\eta})\right| .
\end{aligned}
$$

COROLlaRY 2. If $\mathbf{E} \eta_{k}{ }^{3}|F(\bar{\eta})|<\infty, \mathbf{E} \eta_{j}{ }^{2} \eta_{k}{ }^{2}|F(\bar{\eta})|<\infty$ for all $j, k: j \neq k$, then

$$
\begin{aligned}
& \left|\mathbf{E} F(\bar{\zeta})-\mathbf{E} F(\bar{\eta})+\frac{1}{2} \sum_{j=1}^{n} p_{j}^{2} \mathbf{E} \Delta_{j}^{2} F(\bar{\eta})\right| \\
& \quad \leq \frac{1}{4} \sum_{k=1}^{n} p_{k}^{2} \sum_{j=1}^{k-1} e^{p_{j}} p_{j}^{2} \mathbf{E}\left|\Delta_{j}^{2} \Delta_{k}^{2} F\left(\bar{\psi}_{j}\right)\right|+\frac{1}{3} \sum_{j=1}^{n} e^{p_{j}} p_{j}^{3} \mathbf{E}\left|\Delta_{j}^{3} F\left(\bar{\psi}_{j}\right)\right| .
\end{aligned}
$$

Theorem 1 also allows us to obtain complete asymptotic expansions of $\mathbf{E} F(\bar{\zeta})-\mathbf{E} F(\bar{\eta})$, since the expectations $\mathbf{E} \Delta_{k}^{r} F\left(\bar{\psi}_{k}\right)$ in (3) can be subsequently approximated with expectations $\mathbf{E} \Delta_{k}^{r} F\left(\bar{\eta}_{k}\right)$ using (3).

Corollary 3. Let $l \geq 1$. Suppose

$$
\mathbf{E} \eta_{k_{1}}^{r_{1}} \cdots \eta_{k_{s-1}}^{r_{s-1}} \eta_{k_{s}}^{l+s-r_{1}-\cdots-r_{s-1}}|F(\bar{\eta})|<\infty
$$


for all $1 \leq s \leq l, n \geq k_{1}>k_{2}>\cdots>k_{s} \geq 1,2 \leq r_{1} \leq l, 2 \leq r_{2} \leq l+1-$ $r_{1}, \ldots, 2 \leq r_{s-1} \leq l+s-2-r_{1}-\cdots-r_{s-2}$. Then

$$
\begin{aligned}
& \mid \mathbf{E} F(\bar{\zeta})-\mathbf{E} F(\bar{\eta}) \\
& -\sum^{\prime}(-1)^{s+r_{1}+\cdots+r_{s}}\left(r_{1}-1\right) \cdots\left(r_{s}-1\right) \frac{p_{k_{1}}^{r_{1}} \cdots p_{k_{s}}^{r_{s}}}{r_{1} ! \cdots r_{s} !} \Delta_{k_{1}}^{r_{1}} \cdots \Delta_{k_{s}}^{r_{s}} F(\bar{\eta}) \mid \\
& \leq \sum^{\prime \prime} \frac{e^{p_{k_{s}}}}{\left(1-\tilde{p}_{k_{s}}\right)^{2}}\left(r_{1}-1\right) \cdots\left(r_{s-1}-1\right)\left(l+s-r_{1}-\cdots-r_{s-1}-1\right) \\
& \quad \times \frac{p_{k_{1}}^{r_{1}} \cdots p_{k_{s-1}}^{r_{s-1}} p_{k_{s}}^{l+s-r_{1}-\cdots-r_{s-1}}}{r_{1} ! \cdots r_{s-1} !\left(l+s-r_{1}-\cdots-r_{s-1}\right) !} \\
& \quad \times \mathbf{E}\left|\Delta_{k_{1}}^{r_{1}} \cdots \Delta_{k_{s-1}}^{r_{s-1}} \Delta_{k_{s}}^{l+s-r_{1}-\cdots-r_{s-1}} F\left(\bar{\psi}_{k_{s}}\right)\right|
\end{aligned}
$$

where $\sum^{\prime}$ and $\sum^{\prime \prime}$ denote the following sums:

$$
\begin{aligned}
\sum^{\prime} & =\sum_{s=1}^{l-1} \sum_{k_{1}=1}^{n} \sum_{r_{1}=2}^{l} \sum_{k_{2}=1}^{k_{1}-1} \sum_{r_{2}=2}^{l+1-r_{1}} \cdots \sum_{k_{s}=1}^{k_{s-1}-1} \sum_{r_{s}=2}^{l+s-1-r_{1}-\cdots-r_{s-1}}, \\
\sum^{\prime \prime} & =\sum_{s=1}^{l} \sum_{k_{1}=1}^{n} \sum_{r_{1}=2}^{l} \sum_{k_{2}=1}^{k_{1}-1} \sum_{r_{2}=2}^{l+1-r_{1}} \cdots \sum_{k_{s-1}=1}^{k_{s-2}-1} \sum_{r_{s-1}=2}^{l+s} \sum_{k_{s}=1}^{l+2-r_{1}-\cdots-r_{s-2}} .
\end{aligned}
$$

In the last sum we assume $k_{0}=n+1$ if $s=1$.

Now we consider the Poisson approximation for moments of functions of sums of independent Bernoulli r.v.s. Put $S=\zeta_{1}+\cdots+\zeta_{n}, Z=\eta_{1}+\cdots+\eta_{n}$ and let $h$ be an arbitrary function of nonnegative integer. Introduce the following notation: $\lambda=p_{1}+\cdots+p_{n}, \tilde{p}_{k}=\max \left\{p_{1}, \ldots, p_{k}\right\}, \tilde{p}_{0}=0, \tilde{p}=\tilde{p}_{n}$.

\section{Theorem 2. Let $\mathbf{E}|h(Z)|<\infty$. Then}

$$
\mathbf{E} h(Z)-\mathbf{E} h(S)=\sum_{k=1}^{n} \sum_{r=2}^{\infty} \frac{p_{k}^{r}}{r !} \mathbf{E} \Delta^{r} h\left(T_{k}\right)
$$

where $T_{k}=\zeta_{1}+\cdots+\zeta_{k-1}+\eta_{k+1}+\cdots+\eta_{n}$, and, for each $k$, corresponding series in (4) absolutely converges.

Moreover, if $\mathbf{E} Z^{s+1}|h(Z)|<\infty$ then, first,

$$
\left|\sum_{r=s+1}^{\infty} \frac{p_{k}^{r}}{r !} \mathbf{E} \Delta^{r} h\left(T_{k}\right)\right| \leq \frac{e^{p_{k}}}{\left(1-\tilde{p}_{k-1}\right)^{2}} \frac{p_{k}^{s+1}}{(s+1) !} \mathbf{E}\left|\Delta^{s+1} h(Z)\right|, \quad s \geq 1
$$


and, second, another expansion of the difference $\mathbf{E} h(Z)-\mathbf{E} h(S)$ holds:

$$
\left|\mathbf{E} h(Z)-\mathbf{E} h(S)-\sum_{r=2}^{s}(-1)^{r}(r-1) \sum_{k=1}^{n} \frac{p_{k}^{r}}{r !} \mathbf{E} \Delta^{r} h\left(Y_{k}\right)\right|
$$

$$
\leq \frac{s}{(s+1) !} \sum_{k=1}^{n} \frac{e^{p_{k}}}{\left(1-\tilde{p}_{k-1}\right)^{2}} p_{k}^{s+1} \mathbf{E}\left|\Delta^{s+1} h(Z)\right|,
$$

where $Y_{k}=\zeta_{1}+\cdots+\zeta_{k-1}+\eta_{k}+\cdots+\eta_{n}$.

REMARK 3. The principal distinction between Theorem 1 and Theorem 2 is the appreciably sharper upper bound for the remainder in Theorem 2 which is obtained by the corresponding upper bound for the Radon-Nikodym derivative in Lemma 2 (see Section 2). Formal application of Theorem 1 to functions of sums of the arguments yields an upper bound for the remainder which is substantially rougher than that in Theorem 2 as $\lambda \rightarrow \infty$.

REMARK 4. As noted in Proposition 1, the finiteness of $\mathbf{E}\left|\Delta^{s+1} h(Z)\right|$ is equivalent to finiteness of $\mathbf{E}\left|Z^{s+1} h(Z)\right|$. Nevertheless, the series (4) absolutely converges under weaker $(s=-1)$ moment restrictions.

Corollary 4. If $\mathbf{E} Z^{2}|h(Z)|<\infty$, then

$$
|\mathbf{E} h(S)-\mathbf{E} h(Z)| \leq \frac{1}{2} \frac{e^{\tilde{p}}}{(1-\tilde{p})^{2}} \sum_{j=1}^{n} p_{j}^{2} \mathbf{E}\left|\Delta^{2} h(Z)\right| .
$$

COROLlary 5. If $\mathbf{E} Z^{4}|h(Z)|<\infty$, then

$$
\begin{aligned}
& \left|\mathbf{E} h(S)-\mathbf{E} h(Z)+\frac{1}{2} \sum_{j=1}^{n} p_{j}^{2} \mathbf{E} \Delta^{2} h(Z)\right| \\
& \quad \leq \frac{e^{\tilde{p}}}{(1-\tilde{p})^{2}}\left\{\frac{1}{3} \sum_{j=1}^{n} p_{j}^{3} \mathbf{E}\left|\Delta^{3} h(Z)\right|+\frac{1}{8}\left(\sum_{j=1}^{n} p_{j}^{2}\right)^{2} \mathbf{E}\left|\Delta^{4} h(Z)\right|\right\} .
\end{aligned}
$$

Corollary 6. If $\mathbf{E} Z^{6}|h(Z)|<\infty$, then

$$
\begin{aligned}
& \mid \mathbf{E} h(S)-\mathbf{E} h(Z)+\frac{1}{2} \sum_{j=1}^{n} p_{j}^{2} \mathbf{E} \Delta^{2} h(Z) \\
& \quad-\frac{1}{3} \sum_{j=1}^{n} p_{j}^{3} \mathbf{E} \Delta^{3} h(Z)-\frac{1}{8}\left(\sum_{j=1}^{n} p_{j}^{2}\right)^{2} \mathbf{E} \Delta^{4} h(Z) \mid
\end{aligned}
$$




$$
\begin{aligned}
\leq \frac{e^{\tilde{p}}}{(1-\tilde{p})^{2}}\left\{\frac{1}{4} \sum_{j=1}^{n} p_{j}^{4} \mathbf{E}\left|\Delta^{4} h(Z)\right|\right. & +\frac{1}{6} \sum_{j=1}^{n} p_{j}^{2} \sum_{k=1}^{n} p_{k}^{3} \mathbf{E}\left|\Delta^{5} h(Z)\right| \\
& \left.+\frac{1}{48}\left(\sum_{j=1}^{n} p_{j}^{2}\right)^{3} \mathbf{E}\left|\Delta^{6} h(Z)\right|\right\} .
\end{aligned}
$$

The complete asymptotic expansion for the sums can be written as follows:

Corollary 7. If $\mathbf{E}\left|\Delta^{2 l} h(Z)\right|<\infty$, then

$$
\begin{aligned}
& \mid \mathbf{E} h(S)-\mathbf{E} h(Z) \\
& -\sum^{\prime}(-1)^{s+r_{1}+\cdots+r_{s}}\left(r_{1}-1\right) \cdots\left(r_{s}-1\right) \frac{p_{k_{1}}^{r_{1}} \cdots p_{k_{s}}^{r_{s}}}{r_{1} ! \cdots r_{s} !} \mathbf{E} \Delta^{r_{1}+\cdots+r_{s}} h(Z) \mid \\
& \leq \sum^{\prime \prime} \frac{e^{p_{k_{s}}}}{\left(1-\tilde{p}_{k_{s}}\right)^{2}}\left(r_{1}-1\right) \cdots\left(r_{s-1}-1\right)\left(l+s-r_{1}-\cdots-r_{s-1}-1\right) \\
& \quad \times \frac{p_{k_{1}}^{r_{1}} \cdots p_{k_{s-1}}^{r_{s-1}} p_{k_{s}}^{l+s-r_{1}-\cdots-r_{s-1}}}{r_{1} ! \cdots r_{s-1} !\left(l+s-r_{1}-\cdots-r_{s-1}\right) !} \mathbf{E}\left|\Delta^{l+s} h(Z)\right|,
\end{aligned}
$$

where $\sum^{\prime}$ and $\sum^{\prime \prime}$ denote the following sums:

$$
\begin{aligned}
\sum^{\prime} & =\sum_{s=1}^{l-1} \sum_{k_{1}=1}^{n} \sum_{r_{1}=2}^{l} \sum_{k_{2}=1}^{k_{1}-1} \sum_{r_{2}=2}^{l+1-r_{1}} \cdots \sum_{k_{s}=1}^{k_{s-1}-1} \sum_{r_{s}=2}^{l+s-1-r_{1}-\cdots-r_{s-1}}, \\
\Sigma^{\prime \prime} & =\sum_{s=1}^{l} \sum_{k_{1}=1}^{n} \sum_{r_{1}=2}^{l} \sum_{k_{2}=1}^{k_{1}-1} \sum_{r_{2}=2}^{l+1-r_{1}} \cdots \sum_{k_{s-1}=1}^{k_{s-2}-1} \sum_{r_{s-1}=2}^{l+s-2-r_{1}-\cdots-r_{s-2}} \sum_{k_{s}=1}^{k_{s-1}-1} .
\end{aligned}
$$

In the last sum we suppose $k_{0}=n+1$ if $s=1$.

2. Preliminary results. In this section we prove the following three lemmas, which are also of independent interest.

LEMMA 1. Let $p_{1}=\cdots=p_{n}=p$. Then

$$
\sup _{j} \frac{\mathbf{P}(S=j)}{\mathbf{P}(Z=j)} \leq \frac{1}{1-p}
$$

and, moreover,

$$
\frac{\mathbf{P}(S=j)}{\mathbf{P}(Z=j)} \leq 1
$$

if $j \leq \max \{0, \lambda-\sqrt{2 n(-\log (1-p))}+1\}$ or $j \geq \lambda+\min \{\lambda, \sqrt{2 \lambda}\}+1$. 
PROOF. For $j \leq n$,

$$
\begin{aligned}
\frac{\mathbf{P}(S=j)}{\mathbf{P}(Z=j)} & =\frac{n(n-1) \cdots(n-j+1)}{n^{j}(1-p)^{j}}(1-p)^{n} e^{n p} \\
& =\exp \left\{n(p+\log (1-p))-j \log (1-p)+\sum_{i=0}^{j-1} \log \left(1-\frac{i}{n}\right)\right\} \\
& \leq \exp \{-\log (1-p)+n(p+\log (1-p))-(j-1) \log (1-p) \\
& \left.+n \int_{0}^{(j-1) / n} \log (1-x) d x\right\} \\
& \leq \exp \left\{-\log (1-p)-n H_{p}\left(\frac{j-1}{n}\right)\right\}
\end{aligned}
$$

where $H_{p}(x)=-p+x+(1-x) \log ((1-x) /(1-p))$. The following properties of $H_{p}$ are obvious: $H_{p}(x) \geq 0$ if $x \leq 1$ [hence (7) is true], $H_{p}(1)=1-p, H_{p}(p)=0$, $\frac{d}{d x} H_{p}(p)=0, \frac{d^{2}}{d x^{2}} H_{p}(x)=1 /(1-x)$, that implies

$$
H_{p}(x) \geq \frac{(x-p)^{2}}{2(1-p)} \quad \text { if } p \leq x \leq 1 .
$$

Hence, for $j \geq \lambda+1$,

$$
\frac{\mathbf{P}(S=j)}{\mathbf{P}(Z=j)} \leq \exp \left\{-\log (1-p)-\frac{(j-1-n p)^{2}}{2 n(1-p)}\right\} \leq 1 \quad \text { if } j \geq \lambda+\sqrt{2 \lambda}+1 .
$$

Analogously,

$$
H_{p}(x) \geq \frac{(x-p)^{2}}{2} \quad \text { if } x \leq p .
$$

And hence, for $j \leq \lambda+1$,

$$
\begin{aligned}
\frac{\mathbf{P}(S=j)}{\mathbf{P}(Z=j)} \leq \exp \left\{-\log (1-p)-\frac{(j-1-n p)^{2}}{2 n}\right\} & \leq 1 \\
\quad \text { if } j & \leq \lambda-\sqrt{2 n(-\log (1-p))}+1 .
\end{aligned}
$$

We also have

$$
\begin{aligned}
\frac{\mathbf{P}(S}{\mathbf{P}(Z} & =j) \\
& =\exp \left\{n(p+\log (1-p))-j \log (1-p)+\sum_{i=0}^{j-1} \log \left(1-\frac{i}{n}\right)\right\} \\
& \leq \exp \{n(p+\log (1-p))-j \log (1-p)-1 / n-2 / n-\cdots-(j-1) / n\} \\
& \leq \exp \left\{j n^{-1}(n p-(j-1) / 2)\right\} \leq 1 \quad \text { if } j \geq 2 \lambda+1 .
\end{aligned}
$$

The lemma is proved. 
LEMMA 2. In the case of arbitrary $p_{j}$, the following inequality holds:

$$
\sup _{j} \frac{\mathbf{P}(S=j)}{\mathbf{P}(Z=j)} \leq \frac{1}{(1-\tilde{p})^{2}} .
$$

PROOF. Denote by $S\left(a_{1}, \ldots, a_{m}\right)$ the sum of $m$ independent Bernoulli r.v.s the $j$ th of which is equal to 1 with probability $a_{j}$. Denote also by $Z(b)$ a Poisson r.v. with parameter $b$. Let $g$ be an arbitrary real function. The proof of Corollary 2.1 in Hoeffding (1956) does not have to be changed when it is applied to the similar statement concerning

$$
\sup \left\{\mathbf{E} g\left(S\left(a_{1}, \ldots, a_{n}\right)\right): 0 \leq a_{1} \leq \tilde{p}, \ldots, 0 \leq a_{n} \leq \tilde{p}, a_{1}+\cdots+a_{n}=\lambda\right\} .
$$

This sumpremum is attained with such $a_{1}, \ldots, a_{n}$ that $a_{1}=a_{2}=\cdots=a_{m}=a$, $a_{m+1}=\cdots=a_{k}=\tilde{p}, a_{k+1}=\cdots=a_{n}=0$ for some $a, m, k, 0<a \leq \tilde{p}, 1 \leq m \leq$ $k \leq n$.

Now, for each $j=0,1, \ldots, n$, we set $g(y)=I(y=j)$ and find the corresponding values $a=a(j), m=m(j), k=k(j)$. Let $a_{1}(j)=\cdots=a_{m(j)}(j)=a(j)$, $a_{m(j)+1}(j)=\cdots=a_{k(j)}(j)=\tilde{p}, a_{k(j)+1}(j)=\cdots=a_{n}(j)=0$.

In the case $m(j)<k(j)$ we have

$$
\begin{aligned}
\frac{\mathbf{P}(S=j)}{\mathbf{P}(Z=j)} \leq & \frac{\mathbf{P}\left(S\left(a_{1}(j), \ldots, a_{n}(j)\right)=j\right)}{\mathbf{P}(Z=j)} \\
= & \frac{\mathbf{P}\left(S_{1}\left(a_{1}(j), \ldots, a_{m(j)}(j)\right)+S_{2}\left(a_{m(j)+1}(j), \ldots, a_{k(j)}(j)\right)=j\right)}{\mathbf{P}\left(Z_{1}(m(j) a(j))+Z_{2}((k(j)-m(j)) \tilde{p})=j\right)} \\
\leq & \sup _{i \geq 0} \frac{\mathbf{P}\left(S\left(a_{1}(j), \ldots, a_{m(j)}(j)\right)=i\right)}{\mathbf{P}(Z(m(j) a(j))=i)} \\
& \times \sup _{i \geq 0} \frac{\mathbf{P}\left(S\left(a_{m(j)+1}(j), \ldots, a_{k(j)}(j)\right)=i\right)}{\mathbf{P}(Z((k(j)-m(j)) \tilde{p})=i)} \\
\leq & \frac{1}{(1-\tilde{p})^{2}},
\end{aligned}
$$

where $S_{1}(\cdot), S_{2}(\cdot)$ and $Z_{1}(\cdot), Z_{2}(\cdot)$ denote pairs of independent r.v.s with the corresponding distributions; the last inequality follows from Lemma 1.

In the case $k(j)=m(j)$ the inequality

$$
\frac{\mathbf{P}(S=j)}{\mathbf{P}(Z=j)} \leq \frac{1}{(1-\tilde{p})^{2}}
$$

is also true. The lemma is proved. 
Lemma 3. Let $Z^{\prime}$ be a Poisson r.v. with parameter $\lambda-\delta$, where $0<\delta<\lambda$. Then

$$
\left|\mathbf{E} g\left(Z^{\prime}\right)-e^{\delta} \sum_{j=0}^{m} \frac{(-\delta)^{j}}{j !} \mathbf{E} g(Z+j)\right| \leq \frac{e^{\delta} \delta^{m+1}}{(m+1) !} \mathbf{E}|g(Z+m+1)|,
$$

if $\mathbf{E}|g(Z+m+1)|<\infty$.

ProOF. We have

$$
\begin{aligned}
\mathbf{E} g\left(Z^{\prime}\right) & =e^{\delta} \mathbf{E} g(Z)(1-\delta / \lambda)^{Z} \\
& =e^{\delta} \mathbf{E} g(Z)(1-\delta / \lambda)^{Z} I(Z \leq m)+e^{\delta} \mathbf{E} g(Z)(1-\delta / \lambda)^{Z} I(Z>m) .
\end{aligned}
$$

We assume binomial coefficient $C_{l}^{j}$ to be zero if $j>l$. Consider the first term on the right-hand side of the last relation. We have

$$
\begin{aligned}
\mathbf{E} g(Z)(1-\delta / \lambda)^{Z} I(Z \leq m) & =\mathbf{E} g(Z) \sum_{j=0}^{m} C_{Z}^{j}(-\delta / \lambda)^{j} I(Z \leq m) \\
& =\sum_{j=0}^{m}(-\delta / \lambda)^{j} \mathbf{E} C_{Z}^{j} g(Z) I(Z \leq m) \\
& =\sum_{j=0}^{m} \frac{(-\delta)^{j}}{j !} \mathbf{E} g(Z+j) I(Z \leq m),
\end{aligned}
$$

where the last equality is true because of the identity

$$
\mathbf{E} Z(Z-1) \cdots(Z-j) g(Z)=\lambda^{j} \mathbf{E} g(Z+j) .
$$

On the subset of elementary events $\{Z>m\}$, using Taylor's formula we have

$$
(1-\delta / \lambda)^{Z}=\sum_{j=0}^{m} C_{Z}^{j}(-\delta / \lambda)^{j}+C_{Z}^{m+1}(\delta / \lambda)^{m+1} \theta^{m+1},
$$

where $\theta$ is a function of $\delta, \lambda, Z$ and $m$, such that $|\theta| \leq 1$. Hence

$$
\begin{aligned}
\mathbf{E} g(Z)(1-\delta / \lambda)^{Z} I(Z>m)= & \sum_{j=0}^{m} \frac{(-\delta)^{j}}{j !} \mathbf{E} g(Z+j) I(Z>m) \\
& +\mathbf{E} C_{Z}^{m+1}(\delta / \lambda)^{m+1} \theta^{m+1} g(Z) I(Z>m),
\end{aligned}
$$

where the last summand can be easily estimated:

$$
\begin{aligned}
& \left|\mathbf{E} C_{Z}^{m+1}(\delta / \lambda)^{m+1} \theta^{m+1} g(Z) I(Z>m)\right| \\
& \quad \leq \mathbf{E} C_{Z}^{m+1}(\delta / \lambda)^{m+1}|g(Z) I(Z>m)| r \\
& \quad=\frac{\delta^{m+1}}{(m+1) !} \mathbf{E}|g(Z+m+1)| .
\end{aligned}
$$

Combining relations (8)-(10) we complete the proof. 


\section{Proofs of the main results.}

ProOF OF PROPOSITION 1. First show that (a) implies (b):

$$
\begin{aligned}
\mathbf{E}|g(\tau+l)| & =\sum_{j=l}^{\infty} e^{-\mu} \frac{\mu^{k-l}}{(k-l) !}|g(k)| \\
& \leq \mu^{-l} e^{-\mu} \sum_{k=l}^{\infty} \frac{\mu^{k} k^{l}}{k !}|g(k)| \leq \mu^{-l} e^{-\mu} \mathbf{E} \tau^{l}|g(\tau)| .
\end{aligned}
$$

Analogously, (b) implies (a). It is also clear that (b) implies (c), since $\mathbf{E}|g(\tau+l)|$ $<\infty$ implies $\mathbf{E}|g(\tau+k)|<\infty$ for all $k \leq l$, and hence

$$
\mathbf{E}\left|\Delta^{l} g(\tau)\right|=\mathbf{E}\left|\sum_{k=0}^{l}(-1)^{l-k} C_{l}^{k} g(\tau+k)\right| \leq \mathbf{E} \sum_{k=0}^{l} C_{l}^{k}|g(\tau+k)|<\infty .
$$

Now we show that (c) implies (b) if $l=1$. We have

$$
\begin{aligned}
\mathbf{E}|g(\tau+1)| & =\mathbf{E}\left|g(0)+\sum_{j=0}^{\tau} \Delta g(j)\right| \leq|g(0)|+\mathbf{E} \sum_{j=0}^{\tau}|\Delta g(j)| \\
& =|g(0)|+\sum_{k=0}^{\infty} e^{-\mu} \frac{\mu^{k}}{k !} \sum_{j=0}^{k}|\Delta g(j)|=|g(0)|+e^{-\mu} \sum_{j=0}^{\infty} \sum_{k=j}^{\infty} \frac{\mu^{k}}{k !}|\Delta g(j)| \\
& \leq|g(0)|+e^{\mu} \mathbf{E}|\Delta g(\tau)| .
\end{aligned}
$$

Finally, it is easy to prove equivalence of (b) and (c) using induction on $l$.

PROOF OF THEOREM 1. The proof is substantially based on the Lindeberg method which is contained in the following identity:

$$
\mathbf{E} F(\bar{\eta})-\mathbf{E} F(\bar{\zeta})=\sum_{k=1}^{n}\left(\mathbf{E} F\left(\bar{\phi}_{k}+\eta_{k}{\overline{e_{k}}}\right)-\mathbf{E} F\left(\bar{\phi}_{k}+\zeta_{k} \bar{e}_{k}\right)\right) .
$$

We have

$$
\mathbf{E} F\left(\bar{\phi}_{k}+\zeta_{k} \bar{e}_{k}\right)=\mathbf{E} F\left(\bar{\phi}_{k}\right)+p_{k} \mathbf{E} \Delta_{k} F\left(\bar{\phi}_{k}\right)
$$

For any function $g$, the following equality is well known:

$$
\Delta^{r} g(y)=\sum_{j=0}^{r}(-1)^{r-j} \frac{r !}{(r-j) ! j !} g(y+j)
$$


Thus

$$
\begin{aligned}
\mathbf{E} F\left(\bar{\phi}_{k}+\eta_{k} \overline{e_{k}}\right) & =\sum_{j=0}^{\infty} e^{-p_{k}} \frac{p_{k}^{j}}{j !} \mathbf{E} F\left(\bar{\phi}_{k}+j \overline{e_{k}}\right) \\
& =\sum_{j=0}^{\infty} \sum_{t=0}^{\infty} \frac{(-1)^{t} p_{k}^{j+t}}{t ! j !} \mathbf{E} F\left(\bar{\phi}_{k}+j \overline{e_{k}}\right) \\
& =\sum_{r=0}^{\infty} \sum_{j=0}^{r} p_{k}^{r} \frac{(-1)^{r-j}}{j !(r-j) !} \mathbf{E} F\left(\bar{\phi}_{k}+j{\overline{e_{k}}}\right) \\
& =\sum_{r=0}^{\infty} \frac{p_{k}^{r}}{r !} \mathbf{E} \Delta_{k}^{r} F\left(\bar{\phi}_{k}\right),
\end{aligned}
$$

where the order of summing was changed by Fubini's theorem.

Therefore,

$$
\mathbf{E} F\left(\bar{\phi}_{k}+\eta_{k} \bar{e}_{k}\right)-\mathbf{E} F\left(\bar{\phi}_{k}+\zeta_{k} \bar{e}_{k}\right)=\sum_{r=2}^{\infty} \frac{p_{k}^{r}}{r !} \mathbf{E} \Delta_{k}^{r} F\left(\bar{\phi}_{k}\right) .
$$

The last equality together with (11) proves relation (1).

Now we prove (2). Let $\mathbf{E}\left|\Delta_{k}^{s+1} F(\bar{\eta})\right|<\infty$ and hence $\mathbf{E}\left|\Delta_{k}^{s+1} F\left(\bar{\phi}_{k}\right)\right|<\infty$. Thus we have

$$
\begin{aligned}
& \left|\sum_{r=s+1}^{\infty} \frac{p_{k}^{r}}{r !} \mathbf{E} \Delta_{k}^{r} F\left(\bar{\phi}_{k}+j \overline{e_{k}}\right)\right| \\
& \quad=\left|p_{k}^{s+1} \sum_{r=0}^{\infty} \sum_{j=0}^{r} \frac{p_{k}^{r}}{(r+s+1) !} \frac{(-1)^{r-j} r !}{j !(r-j) !} \mathbf{E} \Delta_{k}^{s+1} F\left(\bar{\phi}_{k}+j \overline{e_{k}}\right)\right| \\
& \quad=\left|p_{k}^{s+1} \sum_{j=0}^{\infty} \sum_{t=0}^{\infty} p_{k}^{j+t} \frac{(-1)^{t}(j+t) !}{(j+t+s+1) ! j ! t !} \mathbf{E} \Delta_{k}^{s+1} F\left(\bar{\phi}_{k}+j \overline{e_{k}}\right)\right| \\
& \quad \leq \frac{p_{k}^{s+1}}{(s+1) !} \sum_{j=0}^{\infty} \frac{p_{k}^{j}}{j !}\left|\sum_{t=0}^{\infty} \frac{(s+1) !(j+t) !}{(j+t+s+1) !} \frac{\left(-p_{k}\right)^{t}}{t !}\right|\left|\mathbf{E} \Delta_{k}^{s+1} F\left(\bar{\phi}_{k}+j \bar{e}_{k}\right)\right| \\
& \leq \frac{p_{k}^{s+1}}{(s+1) !} \sum_{j=0}^{\infty} \frac{p_{k}^{j}}{j !} \mathbf{E}\left|\Delta_{k}^{s+1} F\left(\bar{\phi}_{k}+j \overline{e_{k}}\right)\right| \\
& =e^{p_{k}} \frac{p_{k}^{s+1}}{(s+1) !} \mathbf{E}\left|\Delta_{k}^{s+1} F\left(\bar{\phi}_{k}+\eta_{k} \overline{e_{k}}\right)\right| .
\end{aligned}
$$

So, (2) is true. 
Now we proceed to the proof of (3). If $s=1$ then, by (1) and (2), the relation is true. Consider the case $s \geq 2$. Set

$$
f(j)=f_{k}(j)=\mathbf{E} F\left(\bar{\phi}_{k}+j \overline{e_{k}}\right) .
$$

To prove (3) it suffices to show that, for each $k$,

$$
\begin{aligned}
& \left|\mathbf{E} f\left(\eta_{k}\right)-\mathbf{E} f\left(\zeta_{k}\right)-\sum_{r=2}^{s}(-1)^{r}(r-1) \frac{p_{k}^{r}}{r !} \mathbf{E} \Delta^{r} f\left(\eta_{k}\right)\right| \\
& \leq \frac{s}{(s+1) !} e^{p_{k}} p_{k}^{s+1} \mathbf{E}\left|\Delta^{s+1} f\left(\eta_{k}\right)\right| .
\end{aligned}
$$

In order to prove the last relation we need the expression

$$
1-\sum_{j=2}^{m}(-1)^{j}(j-1) C_{r}^{j}, \quad m \geq 2,
$$

to be calculated. In order to do it we use the identity

$$
\sum_{j=0}^{t}(-1)^{j} C_{i}^{j}=(-1)^{t} C_{i-1}^{t}
$$

and derive that

$$
\begin{aligned}
\sum_{j=2}^{m}(-1)^{j} C_{r}^{j} & =(-1)^{m} C_{r-1}^{m}-1+r, \\
-\sum_{j=2}^{m}(-1)^{j} j C_{r}^{j} & =r \sum_{j=1}^{m-1}(-1)^{j} C_{r-1}^{j}=-(-1)^{m} m C_{r-2}^{m-1}-r .
\end{aligned}
$$

Thus, for $m \geq 2$,

$$
\begin{aligned}
1- & \sum_{j=2}^{m}(-1)^{j}(j-1) C_{r}^{j} \\
& =(-1)^{m}\left(C_{r-1}^{m}-m C_{r-2}^{m-1}\right) \\
& =-(-1)^{m}\left(\frac{(m-1)(r-1) !}{m !(r-1-m) !}+\frac{(r-2) !}{(m-1) !(r-1-m) !}\right) .
\end{aligned}
$$

To prove (14) we show by induction on $m$ that, for all $m=2,3, \ldots, s$, the following relation holds:

$$
\begin{array}{r}
\mathbf{E} f\left(\eta_{k}\right)-\mathbf{E} f\left(\zeta_{k}\right)-\sum_{r=2}^{m}(-1)^{r}(r-1) \frac{p_{k}^{r}}{r !} \mathbf{E} \Delta^{r} f\left(\eta_{k}\right) \\
=\sum_{r=m+1}^{\infty}\left(1-\sum_{j=2}^{m}(-1)^{j}(j-1) C_{r}^{j}\right) \frac{p_{k}^{r}}{r !} \Delta^{r} f(0) .
\end{array}
$$


For $m=2$, by (12) and (13), the equality is true. Now suppose that (16) is valid for some $m \geq 2$. Then, by (12) and (15),

$$
\begin{aligned}
\sum_{r=m+1}^{\infty} & \left(1-\sum_{j=2}^{m}(-1)^{j}(j-1) C_{r}^{j}\right) \frac{p_{k}^{r}}{r !} \Delta^{r} f(0) \\
= & (-1)^{m} m \frac{p_{k}^{m+1}}{(m+1) !} \Delta^{m+1} f(0) \\
& +\sum_{r=m+2}^{\infty}\left(1-\sum_{j=2}^{m}(-1)^{j}(j-1) C_{r}^{j}\right) \frac{p_{k}^{r}}{r !} \Delta^{r} f(0) \\
= & (-1)^{m} m \frac{p_{k}^{m+1}}{(m+1) !} \mathbf{E} \Delta^{m+1} f\left(\eta_{k}\right) \\
& +\sum_{r=m+2}^{\infty}\left(1-\sum_{j=2}^{m+1}(-1)^{j}(j-1) C_{r}^{j}\right) \frac{p_{k}^{r}}{r !} \Delta^{r} f(0),
\end{aligned}
$$

and hence (16) is true for $m+1$. Thus (16) is valid for $m=s$.

Finally, for (14) to be proved, it remains only to estimate the right-hand side of the equality (16) for $m=s$. Because of (15) we have

$$
\begin{aligned}
& \left|\sum_{r=s+1}^{\infty}\left(1-\sum_{j=2}^{s}(-1)^{j}(j-1) C_{r}^{j}\right) \frac{p_{k}^{r}}{r !} \Delta^{r} f(0)\right| \\
& =\left|\sum_{r=s+1}^{\infty}\left(\frac{(s-1)(r-1) !}{s !(r-1-s) !}+\frac{(r-2) !}{(s-1) !(r-1-s) !}\right) \frac{p_{k}^{r}}{r !} \Delta^{r} f(0)\right| \\
& =\mid p_{k}^{s+1} \sum_{r=0}^{\infty} \frac{p_{k}^{r}}{(r+s+1) !}\left(\frac{(s-1)(r+s) !}{s ! r !}+\frac{(r+s-1) !}{(s-1) ! r !}\right) \\
& \quad \times \sum_{j=0}^{r} \frac{(-1)^{r-j} r !}{j !(r-j) !} \Delta^{s+1} f(j) \mid \\
& \left.\quad+\frac{(t+j+s-1) !}{(s-1) !(t+j+s+1) ! t !}\right) \Delta^{s+1} f(j) \mid,
\end{aligned}
$$

where the last expression was derived by changing the order of summing and substituting the variable $t=r-j$. To estimate the expression (17) it suffices to 
note that

$$
\left|\sum_{t=0}^{\infty}(-1)^{t} p_{k}^{t}\left(\frac{(s-1)(t+j+s) !}{s !(t+j+s+1) ! t !}+\frac{(t+j+s-1) !}{(s-1) !(t+j+s+1) ! t !}\right)\right| \leq \frac{s}{(s+1) !},
$$

and hence the expression (17) is not greater than

$$
\frac{s}{(s+1) !} p_{k}^{s+1} \sum_{j=0}^{\infty} \frac{p_{k}^{j}}{j !}\left|\Delta^{s+1} f(j)\right|=\frac{s}{(s+1) !} e^{p_{k}} p_{k}^{s+1} \mathbf{E}\left|\Delta^{s+1} f\left(\eta_{k}\right)\right| .
$$

Therefore, (14) is true and hence (3) holds for $s \geq 2$. The theorem is proved.

ProOF OF COROLlary 2. The assertion follows from the two inequalities below which are easy consequences of Theorem 1:

$$
\begin{aligned}
\left|\mathbf{E} F(\bar{\zeta})-\mathbf{E} F(\bar{\eta})+\frac{1}{2} \sum_{j=1}^{n} p_{j}^{2} \mathbf{E} \Delta_{j}^{2} F\left(\bar{\psi}_{j}\right)\right| & \leq \sum_{j=1}^{n} e^{p_{j}} \frac{p_{j}^{3}}{3} \mathbf{E}\left|\Delta_{j}^{3} F\left(\bar{\psi}_{j}\right)\right|, \\
\left|\sum_{k=1}^{n} p_{k}^{2} \mathbf{E} \Delta_{k}^{2} F\left(\bar{\psi}_{k}\right)-\sum_{k=1}^{n} p_{k}^{2} \mathbf{E} \Delta_{k}^{2} F(\bar{\eta})\right| & \leq \sum_{k=1}^{n} p_{k}^{2} \frac{1}{2} \sum_{j=1}^{k-1} p_{j}^{2} \mathbf{E}\left|\Delta_{j}^{2} \Delta_{k}^{2} F\left(\bar{\psi}_{j}\right)\right| .
\end{aligned}
$$

Corollary 3 is proven by repeated application of relation (3). Theorem 2 is the immediate consequence of Theorem 1 and Lemma 2. The proof of Corollary 5 is analogous to that of Corollary 2. Corollary 7 is proven by repeated application of relation (6). Corollary 6 follows from Corollary 7 for $l=3$.

4. Comparison with predecessors's results. We compare the results of the present paper with the corresponding results of Barbour (1987) and Barbour, Chen and Choi (1995). Corollary 4 will be compared with the following theorem due to Barbour, Chen and Choi (1995):

Theorem A. Let $\mathbf{E} Z^{2}|h(Z)|<\infty$. Then

$$
|\mathbf{E} h(S)-\mathbf{E} h(Z)| \leq \frac{1}{2} C\left(\sum_{j=1}^{n} p_{j}^{2}\right)\left(4 \min \left\{1, \lambda^{-1}\right\} \mathbf{E}|h(Z+1)|+\mathbf{E} \Delta^{2}|h(Z)|\right),
$$

where $C=\max _{k} \sup _{j \geq 0} \frac{\mathbf{P}\left(S-\zeta_{k}=j\right)}{\mathbf{P}(Z=j)}$.

Barbour, Chen and Choi (1995) obtained an upper bound for $C$ which implies, in particular, that $C \leq 2 e^{13 / 12} \sqrt{\pi}$ for $\tilde{p} \leq 1 / 2$.

Corollary 5 will be compared with the following theorem in the same paper. 
Theorem B. Let $\mathbf{E} Z^{4}|h(Z)|<\infty$. Then

$$
\left|\mathbf{E} h(S)-\mathbf{E} h(Z)+\frac{1}{2} \sum_{j=1}^{n} p_{j}^{2} \mathbf{E} \Delta^{2} h(Z)\right| \leq C\left\{\left(\sum_{j=1}^{n} p_{j}^{2}\right)^{2} R_{1}+\left(\sum_{j=1}^{n} p_{j}^{3}\right) R_{2}\right\},
$$

where

$$
\begin{aligned}
R_{1}= & 12 \min \left\{1, \lambda^{-2}\right\} \mathbf{E}|h(Z+2)| \\
& +\frac{1}{3} \min \left\{1, \lambda^{-1}\right\}\left(5 \mathbf{E} \Delta^{2}|h(Z+1)|+\mathbf{E} \Delta^{2}|h(Z)|\right) \\
& +\frac{1}{8} \mathbf{E} \Delta^{4}|h(Z)|, \\
R_{2}= & 2 \min \left\{1, \lambda^{-1}\right\}(\mathbf{E}|h(Z+2)|+\mathbf{E}|h(Z+1)|) \\
& +\frac{1}{3}\left(\mathbf{E} \Delta^{2}|h(Z+1)|+2 \mathbf{E} \Delta^{2}|h(Z)|\right),
\end{aligned}
$$

and the constant $C$ is defined in Theorem $A$.

For functions of at most polynomial growth complete asymptotic expansions were obtained by Barbour (1987). The following statement follows from Theorem 2, Remark 3 on it and equality (2.13) in Barbour (1987):

THEOREM C. Let $l \geq 1, H \geq 0$ and $t \geq 0$. Let $h$ be a real function of integer argument. Suppose that $\lambda \geq 1$ and, for all $y,\left|\Delta^{l} h(y)\right| \leq H\left(1+\lambda^{-t / 2}|y-[\lambda]|^{t}\right)$. Then

$$
\begin{aligned}
& \left|\mathbf{E} h(S)-\mathbf{E} h(Z)+\sum_{s=1}^{l-1} \sum_{[s]} \prod_{j=1}^{s} \frac{1}{r_{j} !}\left(\frac{(-1)^{j} \sum_{i=1}^{n} p_{i}^{j+1}}{j+1}\right)^{r_{j}} \mathbf{E} \Delta^{r_{1}+\cdots+r_{s}+s} h(Z)\right| \\
& \leq K H \max _{(s)}\left\{\lambda^{-k / 2} \prod_{j=1}^{k}\left(\sum_{i=1}^{n} p_{i}^{s_{j}+1}\right)\right\} \leq K H \lambda^{l / 2-1} \sum_{i=1}^{n} p_{i}^{l+1},
\end{aligned}
$$

where $\sum_{[s]}$ denotes the sum over all $\left(r_{1}, \ldots, r_{s}\right) \in\left(\mathbf{Z}^{+}\right)^{s}$ such that $\sum_{j=1}^{s} j r_{j}=s$; $\max _{(s)}$ is taken over

$$
\left\{k \geq 1 ; s_{j} \geq 1,1 \leq j \leq k ; \sum_{j=1}^{k} s_{j}=l\right\}
$$

$K$ is some constant depending only on $l$ and $t$.

But if $\lambda \leq 1$ and $\left|\Delta^{l} h(y)\right| \leq H\left(1+y^{t}\right)$, then

$$
\begin{aligned}
& \left|\mathbf{E} h(S)-\mathbf{E} h(Z)+\sum_{s=1}^{l-1} \sum_{[s]} \prod_{j=1}^{s} \frac{1}{r_{j} !}\left(\frac{(-1)^{j} \sum_{i=1}^{n} p_{i}^{j+1}}{j+1}\right)^{r_{j}} \mathbf{E} \Delta^{r_{1}+\cdots+r_{s}+s} h(Z)\right| \\
& \leq K H \max _{(s)}\left\{\prod_{j=1}^{k}\left(\sum_{i=1}^{n} p_{i}^{s_{j}+1}\right)\right\} .
\end{aligned}
$$


We shall compare the above-mentioned results in the case $\lambda \rightarrow \infty, \tilde{p} \rightarrow 0, h(y)$ being an arbitrary polynomial of order $m \geq 3$.

Define coefficients $K_{h}^{j}$ by the relation

$$
h(y)=\sum_{j=0}^{m} K_{h}^{j} y_{[j]},
$$

where $y_{[j]}$ denotes the so-called $j$ th factorial power of $y: y_{[j]}=y(y-1) \cdots(y-$ $j+1)$. From this we can obtain the simple representation for $\Delta^{k} h$ :

$$
\Delta^{k} h(y)=\sum_{j=k}^{m} j(j-1) \cdots(j-k+1) K_{h}^{j} y_{[j-k]} .
$$

In particular,

$$
\mathbf{E} \Delta^{2} h(Z)=\mathbf{E} \sum_{j=2}^{m} j(j-1) K_{h}^{j} Z_{[j-2]}=\sum_{j=2}^{m} j(j-1) K_{h}^{j} \lambda^{j-2} .
$$

It is easy to see that

$$
\begin{gathered}
\mathbf{E}|h(Z+1)| \sim\left|K_{h}^{m}\right| \lambda^{m}, \\
\mathbf{E}\left|\Delta^{2} h(Z)\right| \sim \mathbf{E} \Delta^{2}|h(Z)| \sim\left|\mathbf{E} \Delta^{2} h(Z)\right| \sim\left|K_{h}^{m}\right| m(m-1) \lambda^{m-2}
\end{gathered}
$$

as $\lambda \rightarrow \infty$. Now we compare the following resulting estimates for $\mid \mathbf{E} h(S)-$ $\mathbf{E} h(Z) \mid$ given by the above-listed results:

Corollary 4: $K_{1} \lambda^{m-2} \sum_{j=1}^{n} p_{j}^{2}$;

Theorem A: $K_{2} \lambda^{m-1} \sum_{j=1}^{n} p_{j}^{2}$;

Theorem C: $K_{3} \lambda^{m-3 / 2} \sum_{j=1}^{n} p_{j}^{2}$;

where $K_{1}, K_{2}, K_{3}$ are some positive constants which depend only on $h$. Note that, in the case under consideration, the constant $H$ in Theorem $\mathrm{C}$ must be of order $\lambda^{m-1}$. We see that in this case the upper bound in Theorem A is rougher than that in Theorem $\mathrm{C}$ and that in Corollary 4.

Comparison of Corollary 5, Theorem B and Theorem $\mathrm{C}$ can be done analogously. We get the following bounds for $\left|\mathbf{E} h(S)-\mathbf{E} h(Z)-\frac{1}{2} \sum_{j=1}^{n} p_{j}^{2} \mathbf{E} \Delta^{2} h(Z)\right|$ :

Corollary 5: $K_{4} \lambda^{m-3} \sum_{j=1}^{n} p_{j}^{3}$;

Theorem B: $K_{5} \lambda^{m-1} \sum_{j=1}^{n} p_{j}^{3}$;

Theorem C: $K_{6}\left(\lambda^{m-3}\left(\sum_{j=1}^{n} p_{j}^{2}\right)^{2}+\lambda^{m-5 / 2} \sum_{j=1}^{n} p_{j}^{3}\right)$;

where $K_{4}, K_{5}, K_{6}$ are constants depending only on $h$. To derive the first two of these three estimates the following inequality was used:

$$
\left(\sum_{j=1}^{n} p_{j}^{2}\right)^{2} \leq \lambda \sum_{j=1}^{n} p_{j}^{3}
$$


PROPOSITION 2. Let $p_{1}=\cdots=p_{n}=p$ and $h(y)$ be a polynomial of order $m \geq 2$. Suppose $\lambda \rightarrow \infty$ and $p \rightarrow 0$. Then

$$
\begin{gathered}
|\mathbf{E} h(S)-\mathbf{E} h(Z)| \sim \frac{1}{2} n p^{2} \mathbf{E}\left|\Delta^{2} h(Z)\right|, \\
\left|\mathbf{E} h(S)-\mathbf{E} h(Z)+\frac{1}{2} n p^{2} \mathbf{E} \Delta^{2} h(Z)\right| \sim \frac{1}{3} n p^{3} \mathbf{E}\left|\Delta^{3} h(Z)\right|+\frac{1}{8} n^{2} p^{4} \mathbf{E}\left|\Delta^{4} h(Z)\right|, \\
\left|\mathbf{E} h(S)-\mathbf{E} h(Z)+\frac{1}{2} n p^{2} \mathbf{E} \Delta^{2} h(Z)-\frac{1}{3} n p^{3} \mathbf{E} \Delta^{3} h(Z)-\frac{1}{8} n^{2} p^{4} \mathbf{E} \Delta^{4} h(Z)\right| \\
\sim \frac{1}{4} n p^{4} \mathbf{E}\left|\Delta^{4} h(Z)\right|+\frac{1}{6} n^{2} p^{5} \mathbf{E}\left|\Delta^{5} h(Z)\right|+\frac{1}{48} n^{3} p^{6} \mathbf{E}\left|\Delta^{6} h(Z)\right| .
\end{gathered}
$$

Thus the bounds in Corollaries 4, 5 and 6 are asymptotically precise.

Proof of Proposition 2. Calculating $\mathbf{E} h(S)$ and $\mathbf{E} h(Z)$ is very simple:

$$
\mathbf{E} h(Z)=\sum_{j=0}^{m} K_{h}^{j} \lambda^{j}, \quad \mathbf{E} S^{m}=\sum_{j=0}^{m} K_{h}^{j} n_{[j]} p^{j},
$$

where coefficients $K_{h}^{j}$ are defined by (19). Thus

$$
\mathbf{E} h(Z)-\mathbf{E} h(S) \sim K_{h}^{m} \frac{m(m-1)}{2} \lambda^{m-1} p .
$$

At the same time the following relation was already noted in (21):

$$
\mathbf{E}\left|\Delta^{2} h(Z)\right| \sim\left|K_{h}^{m}\right| m(m-1) \lambda^{m-2} .
$$

Hence the estimate of Corollary 4 is asymptotically precise.

Now we proceed to proving the exactness of Corollary 5. First, consider the case $h(y)=y_{[m]}$. We have

$$
\begin{aligned}
\mathbf{E} h(Z) & =\lambda^{m}, \\
\mathbf{E} h(S) & =n_{[m]} p \\
& =\lambda^{m}-\frac{m(m-1)}{2} \lambda^{m-1} p+\left(\sum_{i=1}^{m-2} \sum_{j=i+1}^{m-1} i j\right) \lambda^{m-2} p^{2}+O\left(\lambda^{m-3} p^{3}\right), \\
& \frac{1}{2} n p^{2} \mathbf{E} \Delta^{2} h(Z)=\frac{m(m-1)}{2} \lambda^{m-1} p .
\end{aligned}
$$

Hence

$$
\begin{aligned}
\left|\mathbf{E} h(Z)-\mathbf{E} h(S)-\frac{1}{2} n p^{2} \mathbf{E} \Delta^{2} h(Z)\right| & \sim\left(\sum_{i=1}^{m-2} \sum_{j=i+1}^{m-1} i j\right) \lambda^{m-2} p^{2} \\
& =\frac{1}{24} m(m-1)(m-2)(3 m-1) \lambda^{m-2} p^{2} .
\end{aligned}
$$


On the other hand, by (20) we have

$$
\begin{aligned}
& \frac{1}{8} n^{2} p^{4} \mathbf{E}\left|\Delta^{4} h(Z)\right|+\frac{1}{3} n p^{3} \mathbf{E}\left|\Delta^{3} h(Z)\right| \\
& \quad=\left(\frac{1}{8} m(m-1)(m-2)(m-3)+\frac{1}{3} m(m-1)(m-2)\right) \lambda^{m-2} p^{2} \\
& \quad=\frac{1}{24} m(m-1)(m-2)(3 m-1) \lambda^{m-2} p^{2} .
\end{aligned}
$$

So, the assertion is asymptotically precise for $h(y)=y_{[m]}$. It is easy to understand that, because of (19), this assertion is also asymptotically precise for any polynomial of order $m$.

The proof of the exactness of Corollary 6 can be conducted analogously using the following identity:

$$
\sum_{i=1}^{m-3} \sum_{j=i+1}^{m-2} \sum_{k=j+1}^{m-1} i j k=\frac{1}{48} m^{2}(m-1)^{2}(m-2)(m-3)=\frac{1}{4} m_{[4]}+\frac{1}{6} m_{[5]}+\frac{1}{48} m_{[6]} \text {. }
$$

5. The approximation for arbitrary distributions. The content of this section is based on and to a considerable extent repeats the idea of Kchinchine (1933) [cf. Borovkov (1988), Borisov (1993, 1996)]. We apply the results of Section 1 to approximation of vectors of r.v.s with arbitrary, not necessarily Bernoulli, distributions. Let $\xi_{1}, \ldots, \xi_{n}$ be independent r.v.s in an arbitrary measurable Abelian group $\mathcal{A}$ with distributions $Q_{1}, \ldots, Q_{n}$, respectively. The "+" operation in $\mathcal{A}$ is assumed to be measurable. Let $P_{1}, \ldots, P_{n}$ be the accompanying Poisson distributions for $Q_{1}, \ldots, Q_{n}$, respectively, and let $\beta_{1}, \ldots, \beta_{n}$ be independent r.v.s with distributions $P_{1}, \ldots, P_{n}$, respectively. Finally, let $G\left(y_{1}, \ldots, y_{n}\right)$ be an arbitrary measurable function of $n$ arguments in $\mathcal{A}$. We evaluate the difference

$$
\mathbf{E} G\left(\xi_{1}, \ldots, \xi_{n}\right)-\mathbf{E} G\left(\beta_{1}, \ldots, \beta_{n}\right)
$$

when both of the expectations exist.

Denote by $F$ the following expectation:

$$
F\left(k_{1}, \ldots, k_{n}\right)=\mathbf{E} G\left(\tau_{1}^{* k_{1}}, \ldots, \tau_{n}^{* k_{n}}\right),
$$

where $\tau_{j}^{* k}=\tau_{j}^{(1)}+\cdots+\tau_{j}^{(k)}$ is the sum of $k$ independent r.v.s such that each of them has the distribution equal to the conditional distribution of $\xi_{j}$ under the condition $\xi_{j} \neq 0$. All the r.v.s $\tau_{1}^{(1)}, \tau_{1}^{(2)}, \ldots, \tau_{n}^{(1)}, \tau_{n}^{(2)}, \ldots$ are supposed to be independent. Let $p_{j}=\mathbf{P}\left(\xi_{j} \neq 0\right), j=1, \ldots, n$. As in Section $1, \zeta_{1}, \ldots, \zeta_{n}$ denote independent Bernoulli r.v.s with the success probabilities $p_{j}=\mathbf{P}\left(\zeta_{j}=1\right)$, and $\eta_{1}, \ldots, \eta_{n}$ denote independent Poisson r.v.s with parameters $p_{1}, \ldots, p_{n}$, respectively. We have

$$
\mathbf{E} G\left(\xi_{1}, \ldots, \xi_{n}\right)=\mathbf{E} F\left(\zeta_{1}, \ldots, \zeta_{n}\right), \quad \mathbf{E} G\left(\beta_{1}, \ldots, \beta_{n}\right)=\mathbf{E} F\left(\eta_{1}, \ldots, \eta_{n}\right) .
$$

Actually, these identities can be easily deduced from the corresponding results in Khintchine (1933) [cf. Borovkov (1988)]. These relations allow us to apply 
Theorem 1 and its corollaries to the approximation for vectors of independent arbitrarily distributed r.v.s in a measurable Abelian group.

In the case when, for each $j$, the conditional distribution of $\xi_{j}$ under the condition $\xi_{j} \neq 0$ coincides with some distribution $Q$ independent of $j$, and

$$
G\left(y_{1}, \ldots, y_{n}\right)=g\left(y_{1}+\cdots+y_{n}\right),
$$

Theorem 2 and its corollaries can be used. Denote by $\tau_{1}, \tau_{2}, \ldots$ i.i.d. r.v.s with distribution $Q$. For

$$
h(k)=\mathbf{E} g\left(\tau_{1}+\cdots+\tau_{k}\right)
$$

then the equalities

$$
\mathbf{E} G\left(\xi_{1}, \ldots, \xi_{n}\right)=\mathbf{E} h(S), \quad \mathbf{E} G\left(\beta_{1}, \ldots, \beta_{n}\right)=\mathbf{E} h(Z)
$$

hold where $S=\zeta_{1}+\cdots+\zeta_{n}, Z=\eta_{1}+\cdots+\eta_{n}$. In fact, these relations were obtained by Khintchine (1933) [cf. Borisov $(1993,1996)$ ]. It is clear that these representations are equivalent to (22). They reduce the problem of Poisson approximation in an abstract sample space to investigation of closeness of a binomial and the corresponding accompanying Poisson distributions.

EXAMPLE. Let $\xi_{1}, \ldots, \xi_{n}$ be arbitrary r.v.s on the real line. Suppose that, for all $j$, the conditional distributions of $\xi_{j}$ under the condition $\xi_{j} \neq 0$ coincide, and

$$
G\left(y_{1}, \ldots, y_{n}\right)=\left(y_{1}+\cdots+y_{n}\right)^{l} .
$$

Also suppose that $\mathbf{E}\left(\tau_{1}\right)^{l}<\infty$. We have

$$
h(k)=\mathbf{E}\left(\tau_{1}+\cdots+\tau_{k}\right)^{l}=\left(\mathbf{E} \tau_{1}\right)^{l} k_{[l]}+B_{l-1}(k)+\mathbf{E} \tau_{1}^{l} k,
$$

where $B_{j-1}(k)$ is a polynomial of $k$ of order $\leq j-1$ whose coefficients depend only on expectations $\mathbf{E} \tau_{1}, \ldots, \mathbf{E} \tau_{1}^{l-1}$. Hence

$$
\Delta^{2} h(k)=\left(\mathbf{E} \tau_{1}\right)^{l} k_{[l-2]}+B_{l-3}^{\prime}(k),
$$

where $B_{l-3}^{\prime}(k)$ is a polynomial of $k$ of order $\leq l-3$ whose coefficients depend only on $\mathbf{E} \tau_{1}, \ldots, \mathbf{E} \tau_{1}^{l-1}$. Therefore, because of Corollary 4 ,

$$
\begin{aligned}
& \left|\mathbf{E}\left(\xi_{1}+\cdots+\xi_{n}\right)^{l}-\mathbf{E}\left(\beta_{1}+\cdots+\beta_{n}\right)^{l}\right| \\
& \quad \leq \frac{1}{2} \frac{e^{\tilde{p}}}{(1-\tilde{p})^{2}} \sum_{j=1}^{n} p_{j}^{2}\left(\left(\mathbf{E} \tau_{1}\right)^{l-2} \lambda^{l-2}+B_{l-3}^{\prime \prime}(\lambda)\right),
\end{aligned}
$$

where $B_{l-3}^{\prime \prime}(\lambda)$ is a polynomial of $\lambda$ of order $\leq l-3$ with coefficients depending only on $\mathbf{E} \tau_{1}, \ldots, \mathbf{E} \tau_{1}^{l-1}$.

Barbour (1987) obtained complete asymptotic expansions for $\mathbf{E} g\left(\xi_{1}+\cdots\right.$ $\left.+\xi_{n}\right)-\mathbf{E} g\left(\beta_{1}+\cdots+\beta_{n}\right)$ in the case when $\xi_{1}, \ldots, \xi_{n}$ are nonnegative integer 
r.v.s and the function $g$ is of at most polynomial growth. But in the case under consideration, when $g(k)=k^{l}$ and, for all $j$, the conditional distributions of $\xi_{j}$ under the condition $\xi_{j} \neq 0$ coincide, these expansions, in general, don't allow us to separate the parameters $p_{1}, \ldots, p_{n}$ and moments of $\tau_{1}$ as it is done in (23). Such separation in Barbour (1987) is possible only for some simplest classes of distributions but not for arbitrary.

6. Expansions under lesser moment restrictions. In this section, under lesser moment restrictions than those in the theorems and corollaries in Section 1, the asymptotic expansions are studied. However, these expansions may appear inconvenient in case of nonidentically distributed r.v.s. We use the notations $p_{k}$, $\lambda_{k}, \lambda, \bar{\zeta}, \bar{\eta}, \bar{e}_{k}, \Delta_{k}$ that were defined in Section 1. In this section, for the sake of convenience, we also suppose that $p_{k} \neq 0$ for all $k$.

At first we give complete asymptotic expansions for $\mathbf{E} F(\bar{\zeta})-\mathbf{E} F(\bar{\eta})$.

COROLlaRY 8. Let $\mathbf{E} \eta_{k}^{l+1}|F(\bar{\eta})|<\infty$ for all $k$ and some $l \geq 1$. Then

$$
\begin{aligned}
& \left|\mathbf{E} F(\bar{\zeta})-\mathbf{E} F(\bar{\eta})+\sum^{\prime}(-1)^{s} \frac{p_{k_{1}}^{r_{1}} \cdots p_{k_{s}}^{r_{s}}}{r_{1} ! \cdots r_{s} !} \Delta_{k_{1}}^{r_{1}} \cdots \Delta_{k_{s}}^{r_{s}} F\left(\bar{\eta}^{\left(k_{1}, \ldots, k_{s}\right)}\right)\right| \\
& \leq \sum^{\prime \prime} e^{p_{k_{s}}} \frac{p_{k_{1}}^{r_{1}} \cdots p_{k_{s-1}}^{r_{s-1}} p_{k_{s}}^{l+s-r_{1}-\cdots-r_{s-1}}}{r_{1} ! \cdots r_{s-1} !\left(l+s-r_{1}-\cdots-r_{s-1}\right) !} \\
& \times \mathbf{E}\left|\Delta_{k_{1}}^{r_{1}} \cdots \Delta_{k_{s-1}}^{r_{s-1}} \Delta_{k_{s}}^{l+s-r_{1}-\cdots-r_{s-1}} F\left(\bar{\phi}_{k_{s}}^{\left(k_{1}, \ldots, k_{s-1}\right)}+\eta_{k_{s}} \bar{e}_{k_{s}}\right)\right|,
\end{aligned}
$$

where the right-hand side of the inequality is finite, and $\bar{\eta}^{\left(k_{1}, \ldots, k_{s}\right)}=\bar{\eta}-\eta_{k_{1}} \bar{e}_{k_{1}}-$ $\cdots-\eta_{k_{s}}{\overline{k_{s}}}_{\bar{s}}, \bar{\phi}_{k_{s}}^{\left(k_{1}, \ldots, k_{s}\right)}=\bar{\phi}_{k_{s}}-\eta_{k_{1}} \bar{e}_{k_{1}}-\cdots-\eta_{k_{s}}{\overline{k_{s}}}_{k_{s}} ; \sum^{\prime}$ and $\sum^{\prime \prime}$ denote the following sums:

$$
\begin{aligned}
\sum^{\prime} & =\sum_{s=1}^{l-1} \sum_{k_{1}=1}^{n} \sum_{r_{1}=2}^{l} \sum_{k_{2}=1}^{k_{1}-1} \sum_{r_{2}=2}^{l+1-r_{1}} \cdots \sum_{k_{s}=1}^{k_{s-1}-1} \sum_{r_{s}=2}^{l+s-1-r_{1}-\cdots-r_{s-1}}, \\
\sum^{\prime \prime} & =\sum_{s=1}^{l} \sum_{k_{1}=1}^{n} \sum_{r_{1}=2}^{l} \sum_{k_{2}=1}^{k_{1}-1} \sum_{r_{2}=2}^{l+1-r_{1}} \cdots \sum_{k_{s-1}=1}^{k_{s-2}-1} \sum_{r_{s-1}=2}^{l+s-2-r_{1}-\cdots-r_{s-2}} \sum_{k_{s}=1}^{k_{s-1}-1} .
\end{aligned}
$$

In the last sum we put $k_{0}=n+1$ if $s=1$.

This corollary is proven through subsequent application of relations (1) and (2) of Theorem 1.

Further we consider the sums $S=\zeta_{1}+\cdots+\zeta_{n}$ and $Z=\eta_{1}+\cdots+\eta_{n}$. As in Section 1 , put $\tilde{p}_{k}=\max \left\{p_{1}, \ldots, p_{k}\right\}, \tilde{p}=\tilde{p_{n}}$ and let $h$ be an arbitrary real function of integer nonnegative argument. For the sake of convenience we put $\tilde{p}_{0}=0$. The following corollaries are proved by using relations (4) and (5) of Theorem 2: 
Corollary 9. Let $\mathbf{E}\left|\Delta^{3} h(Z)\right|<\infty$. Then

$$
\begin{aligned}
& \left|\mathbf{E} h(S)-\mathbf{E} h(Z)+\frac{1}{2} \sum_{j=1}^{n} p_{j}^{2} \mathbf{E} \Delta^{2} h(Z)\right| \\
& \quad \leq \frac{e^{\tilde{p}}}{(1-\tilde{p})^{2}}\left\{\frac{1}{4} \sum_{k=1}^{n} p_{k}^{2} \sum_{j=1}^{k-1} p_{j}^{2} \mathbf{E}\left|\Delta^{4} h\left(Z^{(k)}\right)\right|+\frac{2}{3}\left(\sum_{k=1}^{n} p_{k}^{3}\right) \mathbf{E}\left|\Delta^{3} h(Z)\right|\right\},
\end{aligned}
$$

where $Z^{(k)}$ is a Poisson r.v. with parameter $\lambda-p_{k}$.

REMARK 5. Because of the obvious upper bound for the corresponding Radon-Nykodim derivative the inequality

$$
\mathbf{E}\left|\Delta^{4} h\left(Z^{(k)}\right)\right| \leq e^{p_{k}} \mathbf{E}\left|\Delta^{4} h(Z)\right|
$$

holds. The right-hand side of the inequality may be infinite while the left-hand side is finite if $\mathbf{E}|h(Z)|<\infty$ and $p_{k} \neq 0$.

PROOF OF COROLlary 9. Because of relations (4) and (5) of Theorem 2, the following inequalities hold:

$$
\begin{aligned}
\left|\mathbf{E} h(Z)-\mathbf{E} h(S)-\frac{1}{2} \sum_{k=1}^{n} p_{k}^{2} \mathbf{E} \Delta^{2} h\left(T_{k}\right)\right| & \leq \frac{e^{\tilde{p}}}{(1-\tilde{p})^{2}} \sum_{k=1}^{n} \frac{p_{k}^{3}}{6} \mathbf{E}\left|\Delta^{3} h(Z)\right|, \\
\left|\sum_{k=1}^{n} p_{k}^{2} \mathbf{E} \Delta^{2} h\left(T_{k}\right)-\sum_{k=1}^{n} p_{k}^{2} \mathbf{E} \Delta^{2} h\left(Z^{(k)}\right)\right| & \leq \sum_{k=1}^{n} p_{k}^{2} \frac{1}{2} \frac{e^{\tilde{p}}}{(1-\tilde{p})^{2}} \sum_{j=1}^{k-1} p_{j}^{2} \mathbf{E}\left|\Delta^{4} h\left(Z^{(k)}\right)\right|, \\
\left|\sum_{k=1}^{n} p_{k}^{2} \mathbf{E} \Delta^{2} h(Z)-\sum_{k=1}^{n} p_{k}^{2} \mathbf{E} \Delta^{2} h\left(Z^{(k)}\right)\right| & =\left|\sum_{k=1}^{n} p_{k}^{2} \sum_{r=1}^{\infty} \frac{p_{k}^{r}}{r !} \mathbf{E} \Delta^{r+2} h\left(Z^{(k)}\right)\right| \\
& \leq \sum_{k=1}^{n} p_{k}^{2} e^{p_{k}} p_{k} \mathbf{E}\left|\Delta^{3} h(Z)\right|,
\end{aligned}
$$

where the proof of the last inequality is analogous to that of inequality (2) in Theorem 1 . The above three inequalities immediately imply the assertion.

We see that the corollary contains lesser restrictions on moments than those in Corollary 5 or Theorem B.

Corollary 10. Let $\mathbf{E}\left|\Delta^{l+1} h(Z)\right|<\infty$ for some $l \geq 1$. Then

$$
\begin{array}{rl}
\mid \mathbf{E} h(S)-\mathbf{E} & h(Z)+\sum^{\prime}(-1)^{s} \frac{p_{k_{1}}^{r_{1}} \cdots p_{k_{s}}^{r_{s}}}{r_{1} ! \cdots r_{s} !} \mathbf{E}^{r_{1}+\cdots+r_{s}} h\left(Z^{\left(k_{1}, \ldots, k_{s}\right)}\right) \mid \\
\leq \sum^{\prime \prime} & \frac{e^{\tilde{p}_{k_{s}}}}{\left(1-\tilde{p}_{k_{s}}\right)^{2}} \frac{p_{k_{1}}^{r_{1}} \cdots p_{k_{s-1}}^{r_{s-1}} p_{k_{s}}^{l+s-r_{1}-\cdots-r_{s-1}}}{r_{1} ! \cdots r_{s-1} !\left(l+s-r_{1}-\cdots-r_{s-1}\right) !} \\
& \times \mathbf{E}\left|\Delta^{l+s} h\left(Z^{\left(k_{1}, \ldots, k_{s-1}\right)}\right)\right|,
\end{array}
$$


where $Z^{\left(k_{1}, \ldots, k_{s}\right)}=Z-\eta_{k_{1}}-\cdots-\eta_{k_{s}}, \sum^{\prime}$ and $\sum^{\prime \prime}$ denote the following sums:

$$
\begin{aligned}
\sum^{\prime} & =\sum_{s=1}^{l-1} \sum_{k_{1}=1}^{n} \sum_{r_{1}=2}^{l} \sum_{k_{2}=1}^{k_{1}-1} \sum_{r_{2}=2}^{l+1-r_{1}} \cdots \sum_{k_{s}=1}^{k_{s-1}-1} \sum_{r_{s}=2}^{l+s-1-r_{1}-\cdots-r_{s-1}}, \\
\sum^{\prime \prime} & =\sum_{s=1}^{l} \sum_{k_{1}=1}^{n} \sum_{r_{1}=2}^{l} \sum_{k_{2}=1}^{k_{1}-1} \sum_{r_{2}=2}^{l+1-r_{1}} \cdots \sum_{k_{s-1}=1}^{k_{s-2}-1} \sum_{r_{s-1}=2}^{l+s-2-r_{1}-\cdots-r_{s-2}} \sum_{k_{s}=1}^{k_{s-1}-1} .
\end{aligned}
$$

In the last sum we suppose $k_{0}=n+1$ if $s=1$.

This corollary is proven by subsequent application of relations (4) and (5).

It was already noted that the right-hand side of the above inequality is finite since, for any $s \geq 1$,

$$
\mathbf{E}\left|\Delta^{l+s} h\left(Z^{\left(k_{1}, \ldots, k_{s-1}\right)}\right)\right|<\infty
$$

if $\mathbf{E}\left|\Delta^{l+1} h(Z)\right|<\infty$.

By Lemma 3, the expectations of functions of $Z^{\left(k_{1}, \ldots, k_{s}\right)}$ can be expressed through expectations of functions of $Z$. However, application of Lemma 3 leads to necessity for enforcing restrictions on the moments.

\section{REFERENCES}

Barbour, A. D. (1987). Asymptotic expansions in the Poisson limit theorem. Ann. Probab. 15 $748-766$.

Barbour, A. D., Chen, L. H. Y. and Choi, K. P. (1995). Poisson approximation for unbounded functions, I: Independent summands. Statist. Sinica 5 749-766.

Barbour, A. D. and Hall, P. (1984). On the rate of Poisson convergence. Math. Proc. Cambridge Philos. Soc. 95 473-480.

Borisov, I. S. (1993). Strong Poisson and mixed approximations of sums of independent random variables in Banach spaces. Siberian Adv. Math. 3 1-13.

BORISOV, I. S. (1996). Poisson approximation of the partial sum process in Banach spaces. Siberian Math. J. 37 627-634.

Borovkov, K. A. (1988). Refinement of Poisson approximation. Theory Probab. Appl. 33 343347.

ChEN, L. H. Y. (1975). An approximation theorem for convolution of probability measures. Ann. Probab. 3 992-999.

Deheuvels, P. and Pfeifer, D. (1986). A semigroup approach to Poisson approximation. Ann. Probab. 14 663-676.

Deheuvels, P., Karr, A., Pfeifer, D. and Serfling, R. (1988). Poisson approximations in selected metrics by coupling and semigroup methods with applications. J. Statist. Plann. Inference 22 1-22.

HoEFFDING, W. (1956). On the distribution of the number of successes in independent trials. Ann. Math. Statist. 27 713-721.

Khintchine, A. (1933). Asymptotische Gesetze der Wahrschaintlichkeitsrechnung. Ergebnisse der Mathematik und ihrer grenzgebiete. Springer, Berlin.

KRUOPIS YU. (1986a). Precision of approximation of the generalized binomial distribution by convolutions of Poisson measures. Lithuanian Math. J. 26 37-49. 
KRUOPIS YU. (1986b). Approximations of distributions of sums of lattice random variables. I, II. Lithuanian Math. J. 26 465-467, 692-704.

LE CAM L. (1960). An approximation theorem for the Poisson binomial distribution. Pacific J. Math. 10 1181-1197.

NovaK, S. Yu. (1998). On the limiting distribution of extrema. Siberian Adv. Math. 8 70-95.

Prohorov, YU. V. (1953). Asymptotic behavior of the binomial distribution. Uspekhi Mat. Nauk 8 135-142 (in Russian).

Roos, B. (1995). A semigroup approach to Poisson approximation with respect to the point metric. Statist. Probab. Letters 24 305-314.

Roos, B. (1998). Metric multivariate Poisson approximation of the generalized multinomial distribution. Theory Probab. Appl. 43 306-315.

Sobolev Institute of Mathematics 630090, NOVOSIBIRSK

RUSSIA

E-MAIL: sibam@math.nsc.ru ruzankin@math.nsc.ru 